sional practice among psychiatrists is a matter which we believe the psychiatrists' own professional body is in a good position to research. Clearly the DHSS agrees with us as they have provided funds. John GunN Chairman, Research Committee

Institute of Psychiatry, De Crespigny Park, Denmark Hill, SE5

\section{INDO-BRITISH EXCHANGES FOR MENTAL HEALTH PERSONNEL}

DEAR SiR,

It may not be generally known that funds are obtainable from UK aid sources to support reciprocal visits by Indian and British mental health professionals wishing to add to their experience in the promotion of new initiatives in mental health care. This can take the form of a series of exchanges of personnel between institutions in the respective countries, or on an individual basis. Preference is given to projects which may improve access to care for hitherto underprivileged groups.

An obvious area of mutual interest is the development of services for minority groups: but British workers may find much to learn from recent measures to improve health (including mental health) services to the rural population in India-which constitutes the great majority of the Indian population. I should be happy to put anyone interested in this field in touch with colleagues in India who would be interested in welcoming visitors or in developing exchange visits with a UK institution.

G. M. Carstairs

National Institute of Mental Health and Neuro-Sciences, Post Bag No. 2779, Bangalore-560029, India.

\section{OVERSEAS PSYCHIATRISTS IN SCOTLAND}

\section{DeAr Sir,}

Although the specific training needs of overseas psychiatrists have previously been briefly described by several authors (Russell \& Walton, 1970; Brook, 1973), the need for a more detailed appraisal is now apparent as the high failure rate in the College Membership examinations for overseas psychiatrists is documented (Hassall \& Trethowan, 1976).

In England and Wales, Brook (1975) reported that $63.2 \%$ of psychiatrists working in the registrar grade were from overseas. As no similar calculation has been made in Scotland, I have carried out a preliminary analysis of data available from the Scottish Home and Health Department.

The main result of this preliminary and 'unofficial' study is that, of the registrars working in adult or child psychiatry units during 1977, 49.3 per cent graduated outside the British Isles, and that, as in England and Wales, the majority of these psychiatrists were working in the large mental hospitals. Indeed, some mental hospitals had only overseas psychiatrists in the registrar grade, whilst some teaching hospitals had none.

These figures cannot be compared precisely with those for England and Wales because the definition of an overseas psychiatrist was slightly different. It is nevertheless fairly clear that the mental health services 'nationwide' depend very largely on overseas graduates.

This being so, their training and examination performance is a crucial issue for both the College and the University Departments. The uneven distribution of overseas psychiatrists has led to the belief that it is the quality of training received that determines their subsequent examination performance.

Nevertheless, experience in Edinburgh shows that even when the training opportunities are similar, overseas psychiatrists still perform less well at examinations, at least during their first years of training. It is likely, therefore, that an improvement in training opportunities, whilst important in itself, may not necessarily improve the pass rate, unless other factors are also taken into consideration. It is also short-sighted to regard this issue as one which will disappear in the next decade as the output from the UK medical schools increases. It is therefore to be hoped that this problem will continue to be looked at from a variety of different vantage points and that controversial issues will not be avoided, nor the need for further research ignored.

In my opinion, an adequate understanding of these very sensitive issues might include (a) an appreciation of 'culture shock' (Brink \& Saunders, 1976) with its subjective pain and disruption; (b) an awareness of the increasing gap that may exist between the amount of undergraduate teaching, particularly in behavioural sciences and psychiatry in UK medical schools when compared with medical schools in Africa or Asia; (c) a familiarity with the Temporary Registration Assessment Board's examination which may discriminate unfairly by its exemption categories between groups of overseas psychiatrists themselves and also between overseas and UK psychiatrists; and (d) an understanding of the psychology of both prejudice and also migration.

These important issues must continue to be 